\title{
Barriers Associated with Presentation Delay among Breast Cancer Patients at Hawassa University Comprehensive and Specialized Hospital, Southern Ethiopia
}

\author{
Wegene Jemebere \\ School of Nursing, Faculty of Health Sciences, College of Medicine and Health Sciences, Hawassa University, \\ Ethiopia \\ Correspondence: Wegene Jemebere School of Nursing, Faculty of Health Sciences, College of Medicine and \\ Health Sciences, Hawassa University, Ethiopia email: jemebere@gmail.com
}

\begin{abstract}
Background: Breast cancer remains the most common cancer and most common cause of cancer-related mortality among women worldwide.

Objectives: The main purpose of this study is to assess barriers resulting in delayed patient presentation to breast cancer care among breast cancer patients under treatment at Hawassa university comprehensive specialized hospital. This study is the first conducted in the study area.

Methods: A hospital- based descriptive cross-sectional study design was done from 1 Jun - 30 December 2017 to answer the study objectives. All delayed 106 women under breast cancer treatment during data collection period were participated in the study. Structured data collection tool which encompass possible barriers in developing countries were applied to collect data from each study subject. Before the data collection, written ethical clearance letter was obtained from IRB of Hawassa university. Data entry was done using EPI Info 3.5.4 and exported to SPSS version 20.0 software package for analysis.

Results: This study revealed that delay was multi-factorial. Of 106 delayed women to breast cancer care, (98.1\%) didn't have knowledge about sign and symptom of breast cancer; (71.7\%) were thinking the breast cancer lump was not dangerous enough to consult expert health professionals and (64.2\%) using alternative medicines like prayer, herbal remedy or traditional healers. There was no significant statistical association found with major causes of delay and socio-demographic characteristics of the women.

Conclusion: A significant percentage of women with breast cancer in Hawassa and nearby are experiencing presentation delay due socio-cultural, economic and health and health related causes. Hence, an intense and focused awareness campaign about breast cancer is needed to educate the general population by Ethiopian cancer association, Federal ministry of health, regional health offices any other concerned body.
\end{abstract}

Keywords: Breast cancer, Barriers to early patient presentation, Ethiopia, Women, Breast cancer care.

\section{Background}

Breast cancer is the most common cancer in women, accounting for $23 \%$ of all female cancers around the globe (D. M. Parkin and L. M. G. Fernandez, 2006). While incidence rates have historically been higher in the developed world, there has been a recent sharp increase in incidence and mortality in the developing world (P. L.
Porter,2009).Breast cancer is the primary cause of cancer death among women globally. It is estimated that over 508, 000 women died in 2011 due to breast cancer worldwide. Although breast cancer is thought to be a disease of the developed world, almost $50 \%$ of breast cancer cases and $58 \%$ of deaths occur in less developed countries (WHO 2014). 
Africa faces potential increases in breast cancer rates as African women adopt western reproductive and dietary behaviors that have been shown to increase the risk of breast cancer (WHO 2014).Hospital records show that in Ethiopia there are more than 200,000 cancer cases per year where cervical \& breast cancers are the top two cancer types having a lion's share for the high women deaths in the country and most women with breast cancer still present very late and treatment outcomes are often not optimal (Ethiopian Cancer Association, 2008). There are many potential barriers that prevent women from seeking treatment upon first noticing breast cancer symptoms. These include economic and logistical barriers, as well as cultural and social factors such as stigma or poor healthcare-seeking behaviors (Anyanwu et al.,2011). Diagnostic delays of 3-6 months are associated with advanced stage of breast cancer and lower survival. Detection and treatment of cancer at an early stage improves the prospects for long-term survival (Richards et al.,2011). One study in sub-Saharan Africa found that $90 \%$ of breast cancer patients presented with stage III or IV of the disease, exhibited a median primary tumor size of $10 \mathrm{~cm}$, and displayed clinically palpable nodal disease and a pattern of disease so advanced that even optimal Western therapy would offer minimal survival value (A. Fregene and L. A. Newman, 2007).

In Ethiopia, there is no previous research was done and presentation delay of breast cancer among Ethiopian particularly Hawassa women is not well documented and still insufficient published data. Therefore, the aim of this study was understanding of these factors to optimize future interventions that prevent patient delay.

\section{Methods}

Study design and setting: A hospital- based descriptive cross-sectional study design was used from 1 Jun - 30 December 2017 among breast cancer patients under treatment at Hawassa University comprehensive specialized hospital. Hawassa is situated at the eastern shore of Lake Hawassa and is located $275 \mathrm{~km}$ to south of Addis Ababa, the capital city of the country. Hawassa University comprehensive specialized hospital is located in south part of Hawassa town. The hospital has been treating patients from South region especially Sidama zone and from neighboring Oromia region and it is the only hospital giving breast cancer treatment in SNNPR. The services were increased gradually during the past time and currently the hospital has around 350 in-patient beds; different service giving units including secondary eye unit, physiotherapy unit, ENT unit, dermatology unit, pathology unit, Caner treatment unit and dental clinic. The hospital is also center for different initiated projects. The vision is to make the hospital a center of training and research for tropical diseases in addition to curative services. The hospital has a center for cancer treatment encompass surgery and breast cancer drugs/ chemotherapy.

Sample size and sampling procedure: No specific sampling strategy was employed in this study, all 106 delayed women under breast cancer treatment from 1 June to 30 December 2017 were considered for the study.Hawassa university comprehensive specialized hospital was selected because it is the only hospital giving breast cancer treatment in SNNPR and neighboring Oromia region.

Data collection tools and procedures: A structured interviewer-administered questionnaire designed by researcher based on reviewed literature which include possible barriers to early breast cancer care in developing countries were modified as Ethiopian context and used to collect data (Ketan Sharma et al.,2011; Liese Pruitt, et al., 2015).Data were collected through face-to-face interview by two BSc nurses working at breast cancer center with data collection experience and who can fluently communicate with the official language. One Physician working at breast cancer center, who had research experience was recruited as supervisor. The questionnaires were prepared by English then it was translated to an official language of the region Amharic then back to English to keep its consistence. The data collectors were trained for two days about the objective and relevance of the study, confidentiality of information, respondent rights, informed consent, and technique of interview and related issues prior to the start of data collection and were closely supervised during the data

www.internationaljournalofcaringsciences.org 
collection. The collected data were reviewed and checked for completeness before data entry.

Statistical analysis: Data entry was done by using EPI Info 3.5.4 and exported to SPSS version 20.0 software package for analysis. We did descriptive analysis to compute proportions for describing the basic characteristics of the study participant and the barriers resulted in presentation delay.

Ethical approval and consent to participate: A written ethical clearance was obtained from the Institutional Review Board at the College of Medicine and Health Sciences of Hawassa University, Hawassa, Ethiopia. Formal letter of cooperation was written to the Hawassa university comprehensive and specialized hospitals. After provision of sufficient information about the purpose of study, a verbal and written consent were obtained from all study participants. Participants were also informed that participation was on voluntary basis and they could withdraw from the study at any time if they were not comfortable about the questionnaire. There was no study participant age below 23 in this study. Confidentiality of information obtained from the study participants was assured by anonymizing the questionnaires.

\section{Operational definitions:}

Barrier: Any factor prevents a woman to early presentation to breast cancer care.

Delay: Patient delay is defined as women delayed more than 3 months from the appearance of a breast cancer symptoms to the consultation of expert healthcare professional.

\section{Results}

Socio-demographic characteristics of the study participants

A total of 106 women delayed to breast cancer care were participated to the study with (100\%) response rate. Age group of 35-39 were (26.4\%) and married (95.3\%) had the largest proportion. Patients who received elementary school education (29.2\%) had the largest proportion, only (13.2\%) of the participants had history of breast cancer in the family and $(64.2 \%)$ were residents of urban. House wife accounts (42.5\%) and (37.7\%) were
Oromo by ethnicity. Almost all of the respondents $(92.5 \%)$ claimed low economic status (Table 1$)$.

Socio-cultural barriers: The major reason which made women late to early breast cancer care in this study was lack of knowledge about sign and symptom of breast cancer (98.1\%). Even if there is no significant statistical association, lack of knowledge was found to have association with educational status of the women as those who achieved elementary school, high school and college and above were $29.8 \%, 24.0 \%$ and $18.3 \%$ respectively. More than half (64.2\%) of mothers delayed using alternative medicines like prayer, herbal remedy or traditional healers. Using alternative medicine also associated with educational status as those who achieved elementary school, high school and college and above were $34.2 \%, 26.5 \%$ and $17.6 \%$ respectively but it was not statistically significant. Half (51.9\%) of them were late due to fear of examination and treatment like disfigurement after surgery and only (7.5\%) of study participants were late due to stigma of the disease. A large proportion (71.7\%) of them were delayed because they think the breast cancer lump was not dangerous enough to consult expert health professionals and it also associated with educational status as those who achieved elementary school, high school and college and above were $32.9 \%, 21.1 \%$ and $13.2 \%$ respectively but it was not statistically significant. Only (31.1\%) of study participants were late due to lack of social support and it was associated with income of women as $100 \%$ of the them were with low income but is was not statistically significant (Figure 1).

Economic barriers: A great proportion of women (63.2\%) were late because of fear of high cost of medical care and it was associated with income of study participants as $100 \%$ of them were with low income but it was not statistically significant. Only (27.4\%) were late due to high cost of travel to the hospital and almost half (46.2\%) of them due to obligation at home/work. Obligation at home/work also associated with occupation of the women as (38\%) were professional employee but is was not statistically significant (Figure 2). 
Table 1: Socio-demographic characteristics of the women under breast cancer treatment at Hawassa University specialized and comprehensive hospital, Hawassa, SNNPR, Ethiopia, (n=106).

\begin{tabular}{|c|c|c|c|}
\hline \multicolumn{2}{|c|}{ Socio-demographic characteristics } & \multirow{2}{*}{$\begin{array}{l}\text { Frequency } \\
2\end{array}$} & \multirow{2}{*}{$\begin{array}{r}\text { Percent } \\
1.9\end{array}$} \\
\hline \multirow{11}{*}{ Age } & $15-24$ & & \\
\hline & $25-29$ & 4 & 3.8 \\
\hline & $30-34$ & 6 & 5.7 \\
\hline & $35-39$ & 28 & 26.4 \\
\hline & $40-44$ & 14 & 13.2 \\
\hline & $45-49$ & 15 & 14.2 \\
\hline & $50-54$ & 10 & 9.4 \\
\hline & $55-59$ & 13 & 12.3 \\
\hline & $60-64$ & 10 & 9.4 \\
\hline & $65-65+$ & 4 & 3.8 \\
\hline & Total & 106 & 100.0 \\
\hline \multirow{3}{*}{ Marital status } & Married & 101 & 95.3 \\
\hline & Single & 5 & 4.7 \\
\hline & Total & 106 & 100.0 \\
\hline \multirow{5}{*}{$\begin{array}{l}\text { Educational } \\
\text { Status }\end{array}$} & Un educated & 30 & 28.3 \\
\hline & Elementary school & 31 & 29.2 \\
\hline & High school & 25 & 23.6 \\
\hline & College and above & 20 & 18.9 \\
\hline & Total & 106 & 100.0 \\
\hline \multirow{3}{*}{$\begin{array}{l}\text { Breast cancer } \\
\text { in the family }\end{array}$} & Yes & 14 & 13.2 \\
\hline & No & 92 & 86.8 \\
\hline & Total & 106 & 100.0 \\
\hline \multirow{3}{*}{ Residence } & Urban & 68 & 64.2 \\
\hline & Rural & 38 & 35.8 \\
\hline & Total & 106 & 100.0 \\
\hline \multirow{6}{*}{ Occupation } & Professional employ & 31 & 29.2 \\
\hline & Farmer & 3 & 2.8 \\
\hline & Merchant & 17 & 16.0 \\
\hline & House wife & 45 & 42.5 \\
\hline & Daily Laborer & 10 & 9.4 \\
\hline & Total & 106 & 100.0 \\
\hline \multirow{10}{*}{ Ethnicity } & Sidama & 12 & 11.3 \\
\hline & Oromo & 40 & 37.7 \\
\hline & Amhara & 37 & 34.9 \\
\hline & Wolaita & 6 & 5.7 \\
\hline & Tigre & 1 & 0.9 \\
\hline & Gamo & 3 & 2.8 \\
\hline & Silte & 3 & 2.8 \\
\hline & Derashe & 3 & 2.8 \\
\hline & Dawro & 1 & 0.9 \\
\hline & Total & 106 & 100.0 \\
\hline \multirow{3}{*}{ Income } & Low & 98 & 92.5 \\
\hline & Middle & 8 & 7.5 \\
\hline & Total & 106 & 100.0 \\
\hline
\end{tabular}




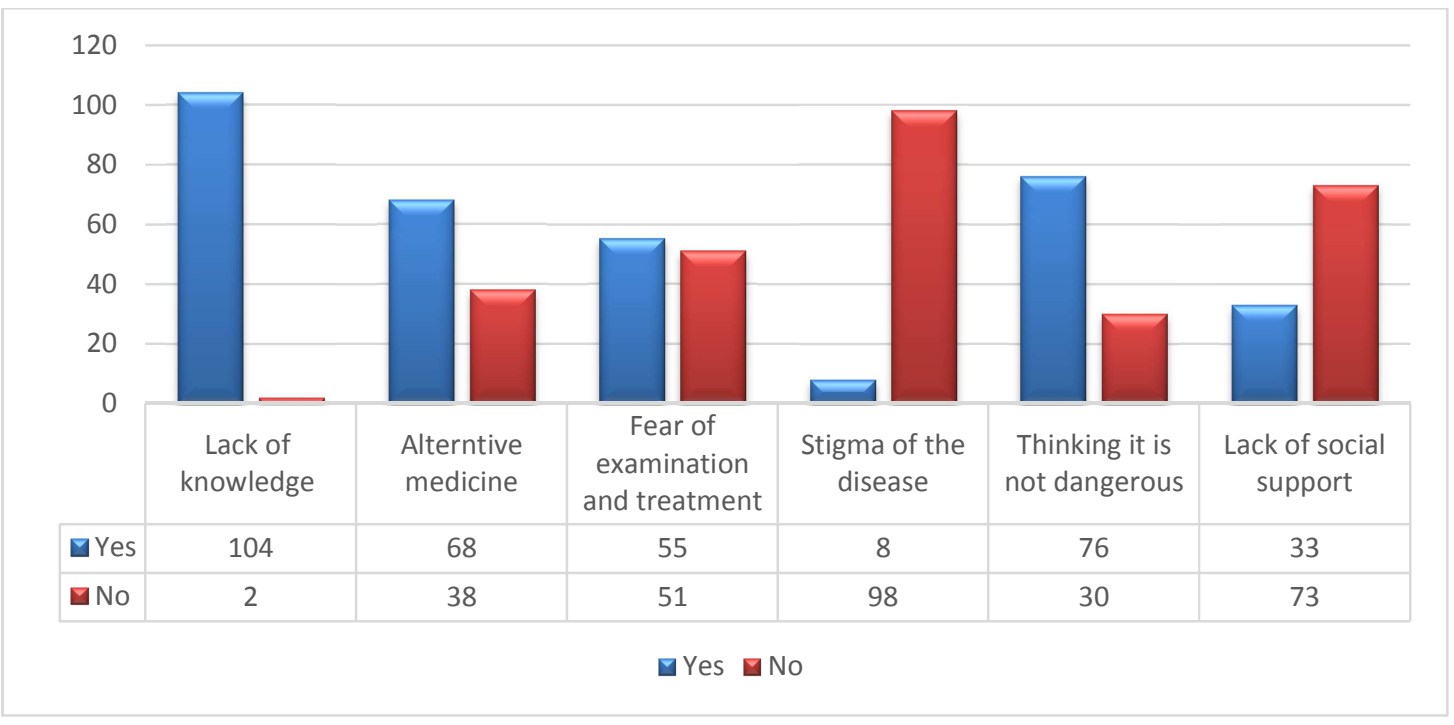

Figure 1: Socio-cultural barriers causing presentation delay to breast cancer care at Hawassa University comprehensive and specialized hospital, Hawassa, South Ethiopia (n=106).

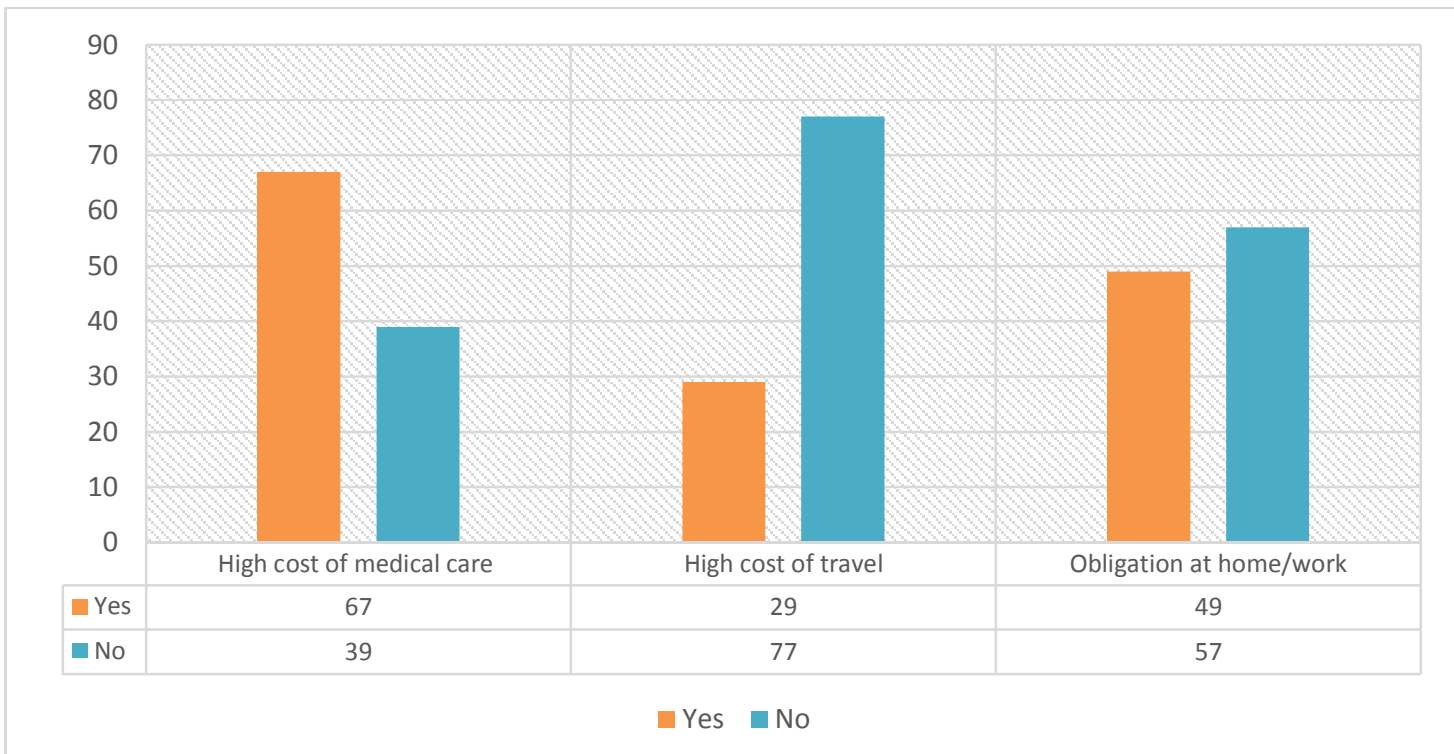

Figure 2: Economic barriers causing presentation delay to breast cancer care at Hawassa University comprehensive and specialized hospital, Hawassa, South Ethiopia $(n=106)$.

Health and health-related barriers: Thirty-two $(30.2 \%)$ of the study participants were late due to inaccessibility health care system and it was associated with income of study participants as $100 \%$ of the them were with low income but it was not statistically significant. A small proportion of women $(18.9 \%)$ were late due to misdiagnosis of the breast cancer as they were informed nothing to worry about the lump by health professionals (Table 2). 
Table 2: Health and health-related barriers causing presentation delay to a breast cancer care at Hawassa university specialized and comprehensive hospital, Hawassa, South Ethiopia ( $n=106)$

\begin{tabular}{lcc}
\hline Health and health-related factors & Yes & No \\
\hline Inaccessibility to health care system & $32(30.2 \%)$ & $74(69.8 \%)$ \\
Misdiagnosis by health professionals & $20(18.9 \%)$ & $86(71.1 \%)$ \\
\hline
\end{tabular}

\section{Discussion}

This study revealed that delay is multi-factorial as socio-cultural, economic as well health and health related factors brought the women late to early breast cancer care. Breast cancer in low-resource countries, little public awareness of cancer generally and breast cancer specifically, are a crucial obstacle where breast cancer commonly remains undiagnosed until it is late stage or metastatic, when treatment options have less benefit or are simply unavailable (Benjamin et al., 2011).

\section{Socio-cultural barriers}

This study revealed that the number one factor brought the affected women late to early breast cancer care was lack of knowledge about sign and symptom of the disease $(98.1 \%)$. This result is much higher than a study done in UK among black women (16\%) [11]. The higher finding in this study may be due to many reasons especially little public awareness of breast cancer in Ethiopia and difference in educational status as a greater proportion of the patients under the study were uneducated. Misconceptions about the nature of breast cancer can lead women to seek alternative care instead of standard treatment (Benjamin et al., 2011). According to this study, the second reason which made the women late was using alternative medicines like prayer, herbal remedy or traditional healers $(64.2 \%)$. This finding is higher than the study conducted in northern Pakistan (40.7\%) (Muhammad Aleem Khan, SehrishShafique, et al., 2015) and the difference may be due to breast cancer is a new problem in Ethiopia and most Ethiopian society believed that cancer is treated by pray, holy water and herbal remedy rather than modern medicines. Examination and treatment fear prolong delay among some women with cancer. In this study, (51.9\%) women were late due to fear of examination and treatment like disfigurement after breast surgery. The finding revealed similar result as $(57 \%)$ of African-American women believed treatments for breast cancer were worse than the disease itself (Jones CEL et al., 2015).

This study revealed that, only $(7.5 \%)$ of women was late because of stigma of the disease. The finding was low as stigma and taboo emerged as salient themes among black women in the USA and the UK (Ketan Sharma et al.,2011). The difference is may be due to culture difference as there is no deep-rooted stigma for cancer in Ethiopian society like sexual transmitted disease like HIV/AIDS. Many of women with cancer had underestimated the significance of their symptoms which contributed to them a delay in presentation (Jones CEL et al., 2015). This study identified that, (71.7\%) of them was late thinking the breast cancer lump was not dangerous enough to consult expert health professionals. This finding was much higher than the study conducted in northern Pakistan (17.1 \%) (Muhammad Aleem Khan, SehrishShafique, et al., 2015) and another study suggested that (13.9\%) African-American women were more likely than white women to delay presentation if their lump was not bothering them (Jones CEL et al., 2015). The higher finding in this study may be due to the fact that a lump caused by breast cancer is not well known and may be perceived other breast problem by Ethiopian women as disease is new and not well promoted by federal ministry of health of Ethiopia. Thirty-three (31.1\%) of study participants were late due to lack of social support. The finding was similar as

www.internationaljournalofcaringsciences.org 
limited evidence indicates that African-American women are more likely than white women to delay seeking help due to lack of partner support (Jones CEL et al., 2015).

\section{Economic barriers}

Even in wealthy societies, women with fewer financial resources are more likely to delay seeking medical attention [8]. In this study, a great proportion of women $(63.2 \%)$ were late due fear of high cost of medical care. The finding was much higher than the study done in USA as (17\%) reported a self-delay which was associated with poorer financial status (Ketan Sharma et al.,2011). The higher finding in this study may be due to the fact that $(92.5 \%)$ of the study participants were low in economic status and most Ethiopian women live below poverty line. This study identified that, almost half $(46.2 \%)$ of women were late due to obligation at home/work. The finding was higher than study done in UK as black women (32\%) and white women $(37 \%)$ to report having too many other things to worry about, and similar proportions reported being too busy to make time to see the medical doctors (Ketan Sharma et al.,2011). The difference is may be due to most Ethiopian women are busy at home and work place to help their husband to earn money.

\section{Health and health-related barriers}

According to this study, (30.2\%) of women were late due to inaccessibility of health care system. The finding is higher than study done USA as the effect of access issues on delay was mixed (Jones CEL et al., 2015). It is clear that accessibility issue is very common in Ethiopia for example Hawassa University comprehensive specialized hospital is the only hospital gives breast cancer treatment in south region of Ethiopia serving more 20 million people. This study showed that, (18.9\%) of women were late due misdiagnosis of the breast cancer by health professionals as they were informed it is nothing to worry about the lump. The finding is higher compared to study done USA as only three African-American women from 210 with cancer were initially told their symptoms were benign and this contributed to delayed diagnosis (Ketan Sharma et al.,2011). The difference is may be due to inadequate number of health professionals specialized in cancer diagnosis and treatment in Ethiopia.

\section{Conclusion}

A significant percentage of women with breast cancer in Hawassa and nearby are experiencing presentation delay due socio-cultural, economic and health and health related factors. This study found strong evidence for lack of knowledge about sign and symptom of breast cancer relating to patient delay as a number one cause. Thinking the breast cancer lump was not dangerous enough to consult expert health professionals; Using alternative medicines like prayer, herbal remedy or traditional healers; fear of high cost of medical care; fear of examination and treatment and obligation at home/work contributed more for delay in this study respectively. Stigma of the disease; misdiagnosis of the breast cancer by health professionals; high cost of travel to the hospital; inaccessibility of health care system and lack of social support contributed less for delay in this study respectively. Hence, an intense and focused awareness campaign is needed to educate the general population about breast cancer by Ethiopian cancer association, Federal ministry of health, regional health offices any other concerned body.

\section{Limitation of the study}

All possible barriers resulting in delay of women to breast cancer center may not be addressed.

\section{List of abbreviations}

WHO: World Health Organization; SBE: Self Breast Examination; BSc: Bachelor of Science; LMIC: Low- to Middle-Income Countries; UK: United Kingdom; USA: United States of America; IRB: Institutional review board of Hawassa University; SNNPRS: Southern Nations Nationalities and Peoples Region; NORAD: Norwegian Agency for Development Cooperation

\section{Declaration}

\section{Ethics approval and consent to participate}

A written ethical clearance was obtained from the Institutional Review Board at the College of Medicine and Health Sciences of Hawassa 
University, Hawassa, Ethiopia. Formal letter of cooperation was written to the Hawassa university comprehensive and specialized hospitals.

After provision of sufficient information about the purpose of study, a verbal and written consent were obtained from all study participants. Participants were also informed that participation was on voluntary basis and they can withdraw from the study at any time if they are not comfortable about the questionnaire. There was no study participant age below 23 in this study. Confidentiality of information obtained from the study participants was assured by anonymizing the questionnaires.

Availability of data and materials: All data generated or analyzed during this study are included in this published article [and its supplementary information files]. We send all which are available; there is no remaining data and materials.

Funding and sponsor ship: This research was funded by NORAD. The role of the funding body was to expense the cost for data collection, analysis and interpretation. The study design and manuscript were designed and written by authors.

Acknowledgement: First of all, I would like to thank office of the vice president for research and technology transfer of Hawassa University for the initiation and arrangement to the development of this study. NORAD also deserves thanks for funding this research. I am also thankful to all women who participated in this study for their commitment in responding to our interviews. Also, I would like to express my deepest gratitude to Dr. Dejene Hailu for providing me essential information.

\section{References}

A. Fregene and L. A. Newman, "Breast cancer in subSaharan Africa: how does it relate to breast cancer in African-American women?" Cancer, vol. 103, no. 8, pp. 1540-1550, 2005.

Anyanwu, S. N. C., Egwuonwu, O. A., \& Ihekwoaba, E. C. (2011). Acceptance and adherence to treatment among breast cancer patients in eastern Nigeria. The Breast, 20 (Suppl. 2), 1-3.

Benjamin O Anderson, Eduardo Cazap, (2011) Optimization of breast cancer management in lowresource and middle-resource countries: executive summary of the Breast Health Global Initiative consensus, Lancet Oncol; 12: 387-98.

D. M. Parkin and L. M. G. Fernandez, "Use of statistics to "assess the global burden of breast cancer," Breast Journal, vol. 12, supplement 1, pp. S70-S80, 2006.

Ethiopian Cancer Association. Fight against tobacco to reduce the Risk of cancer through Anti-tobacco youth clubs in Ethiopia. 2008. Available at: http://www.yeeca.org/Projects.htm.

Jones CE, Maben J, Jack RH, Davies EA, Forbes LJ, Lucas G, Ream E. A systematic review of barriers to early presentation and diagnosis with breast cancer among black women. BMJ Open 2014;4: e004076. doi:10.1136/bmjopen-2013-004076.

Ketan Sharma Ainhoa CostasLawrence N Shulman. (2012) A Systematic Review of Barriers to Breast Cancer Care in Developing Countries Resulting in Delayed Patient Presentation. Hindawi Publishing Corporation, Journal of Oncology, (8):121873

Liese Pruitt, Tolulope Mumuni, Eugene Raikhel, Adeyinka Ademola, Temidayo Ogundiran, Adeniyi Adenipekun, Imran Morhason-Bello Oladosu A. Ojengbede \& Olufunmilayo I. (2015) Social barriers to diagnosis and treatment of breast cancer in patients presenting at a teaching hospital in Ibadan, Nigeria, Global Public Health: An International Journal for Research, Policy and Practice, 10:3, 331344 ,

Muhammad Aleem Khan, SehrishShafique, et al (2015). Presentation Delay in Breast Cancer Patients, Identifying the Barriers in North Pakistan. Asian Pac J Cancer Prev, 16 (1), 377-380.

P. L. Porter, "Global trends in breast cancer incidence and mortality," Salud Publica de M exico, vol. 51, supplement 2, pp. s141-s146, 2009.

Richards, M., Smith, P., Ramirez, A., Fentiman, I., \& Rubens, R. (2009). The influence on survival of delay in the presentation and treatment of symptomatic breast cancer. British Journal of Cancer, 79, 858-864. doi: 10.1038/sj.bjc.6690137.

World health organization. Breast cancer: prevention and control. WHO 2014. Available at: http://www.who.int/entity /cancer/detection/en/ 